\title{
Compared Between Ipv6 And With Ipv4,Differences And Similarities
}

\author{
Z. Z. AbouSalem ${ }^{(1)}$, M. A. Ashabrawy ${ }^{(2)}$ \\ (1), (2) Prince Sattam binAbdulaziz University, Computer Department, KSA \\ (2) Reactors Department, Computer Science, Nuclear Research Center, Atomic Energy Authority Egypt. \\ deeb2222@hotmail.com, ashabrawy@hotmail.com
}

\begin{abstract}
This paper provides Advantages of using IPv6 and comparison of IPv4 and IPv6,For that, it's critical to understand the differences and similarities, Some points in the near future when the sheer size of billions new devices will throw the IPv6 switch.IPv4 and IPv6, where IPv6 is the enhanced version of IPv4, There are various differences between IPv4 and IPv6 protocol including its features, but the critical one is the number of addresses (Address space) it creates. In my paper, I will explained the main Advantages (differences between both protocols)of IPv6 \& IPv4, By giving results that can be obtained through the diffusion of technology.
\end{abstract}

Keywords: Advantages of using IPv6, IPv4 and IPv6

Language: English

Date of Publication: 29-10-2018

DOI: $10.24297 /$ ijct.v\%vi\%i.7805

ISSN: $2277-3061$

Volume: 17 Issue: 02

Journal: International Journal of Computers \& Technology

Website: https://cirworld.com

This work is licensed under a Creative Commons Attribution 4.0 International License.

How to Cite: Abou Salem, Z. Z., \& A shabrawy, M. A. (2018). Compared between IPv6 and with IPv4, differences and similarities. International Journal Of Computers \& Technology, 17(2), 7340-7348. Retrieved from https://cirworld.com/index.php/ijct/article/view/7805 


\section{Introduction}

The IPv4 address contains approximately 4.3 billion addresses and is managed and distributed by the Internet Assigned Numbers Authority (IANA) to the Internet Registries(RIRs) in blocks of up to 16.8 million addresses each. IP version 4 (IPv4) was started in 2011 for a set of unallocated addresses [1]. This depletion led to research and development to the next successor which is Internet Protocol Version 6 (IPV6) [2]. The new version of Internet Protocol 6 (IPv6) is the technology that is designed to address the problem. IPV6 supports approximately $3.4 \times 10^{38}$ network address which translates to the equivalent of 340 trillion address numbers, ie about 670 quadrillion address per square millimeter of the Earth's surface[3] and [4].

This paper provides information on why IP version 6 (IPv6) becomes the Internet standard instead of Internet Protocol version 4 (IPv4), and what features this protocol has.IPv6 is the next generation of IP protocol. Most computers on the Internet use the IPv4 protocol, which has been considered quite reliable and flexible for 20 years [5]. However, due to the growth of the Internet network, the IPv4 protocol is becoming less convenient due to the limitations it provides. IPv6 is an updated version of IPv4, which gradually replaces the latter as an Internet standard.Most engineers are running IPv4 and IPv6 together. It does so by creating a new version of the protocol which serves the function of IPv4, but without the same limitations of IPv4. IPv6 is not totally different from IPv4: what you have learned in IPv4 will be valuable when you deploy IPv6 [6] and [7].

The main characteristics of this protocol had to be the following:

1. Larger addressing space, structured addresses andno addresses classes.

2. Automatic configuration.

3. Simplified routing.

4. Better structuring options for the networks.

5. Improved security features.

6. Support for real-time and multimedia services

\section{Internet Protocol Version4 (IPV4)}

An IPv4 address is a 32-bit address that is usually represented in dotted decimal notation, with a decimal value representing each of the four octets (bytes) that make up the address [8]. For example:

$\begin{array}{cccc}00001001 & 01000011 & 01100001 & 00000010 \\ 00001001 & 01000011 & 01100001 & 000000104 \text { octets }\end{array}$

9

$67 \quad 97$

2

dotted decimal

notation (9.67.97.2)

The IPv4 address consists of a network address and a host address. Within the Internet, the network addresses are assigned by a central authority, the Network Information Center (NIC). The portion of the IPv4 address that is used for each of these addresses is determined by the class of address. There are three commonly used classes of IPv4 addresses (see Figure 1) [9].

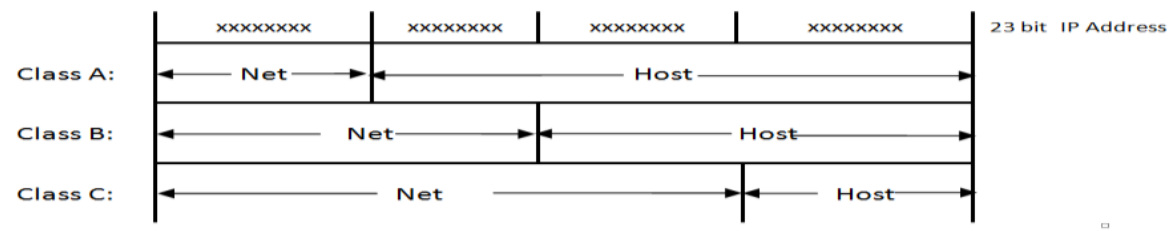

Figure 1. Classes of IPv4 addresses 
The class of the address is determined by the first octet of the IPv4 address. Figure 2 shows how the class of address is determined. The figure also shows Class D addresses. Class D addresses represent multicast groups, not network IP addresses. Multicast group addresses consist of the high-order, four bits of 1110 and the remaining 28 bits, which form a multicast group ID[9] and [10].

\begin{tabular}{|c|c|c|c|c|c|}
\hline \multicolumn{2}{|c|}{23 bit address } & $x x x x x x x x$ & $x x x x x x x x$ & $x X X X X X X X$ & $x X X X X X X X$ \\
\hline \multirow[t]{4}{*}{ Class A } & min max rang & $0 x x x x x x x$ & $x x x x x x x x$ & $x x x x x x x x$ & $x x x x x x x x$ \\
\hline & & 00000000 & & & \\
\hline & & 01111111 & & & \\
\hline & & $1-126$ & (decimal notation; & and 127 ar & reserved \\
\hline \multirow[t]{4}{*}{ Class B } & min max rang & $10 x x x x x x$ & $x x x x x x x x$ & $x x x x x x x x$ & $x x x x x x x x$ \\
\hline & & 10000000 & & & \\
\hline & & 10111111 & & & \\
\hline & & $128-191$ & (decimal notation) & & \\
\hline \multirow[t]{4}{*}{ Class C } & min max rang & $110 x x x x x$ & $x X X X X X X X$ & $x x x x x x x x$ & $x X X X X X X X$ \\
\hline & & 11000000 & & & \\
\hline & & 11011111 & & & \\
\hline & & $192-223$ & (decimal notation) & & \\
\hline \multirow[t]{4}{*}{ Class D } & min max rang & $1110 x x x x x$ & $x x x x x x x x$ & $x x x x x x x x$ & $x X X X X X X X$ \\
\hline & & 11100000 & & & \\
\hline & & 11101111 & & & \\
\hline & & $224-239$ & (decimal notation) & & \\
\hline
\end{tabular}

Figure 2. Determining the class of an IPv4 address

As shown in Figure 2, the value of the bits in the first octet determine the class of address, and the class of address determines the range of values for the network and host segment of the IPv4 address. For example, the IPv4 address 9.67.97.2 would be a class A address, since the first two bits in the first octet contain B'00'. The network part of the IPv4 address is 9 and the host part of the IPv4 address is 67.97.2. But despite these measures the IPV4 addresses are being consumed at an alarming rate Primary reason is huge growth in number of internet users,mobile devices using Internet connection and always on devices such as ADSL modems and cable modems. This brings us to the development and adoption of IPV 6 as an alternate solution [4] and [11].

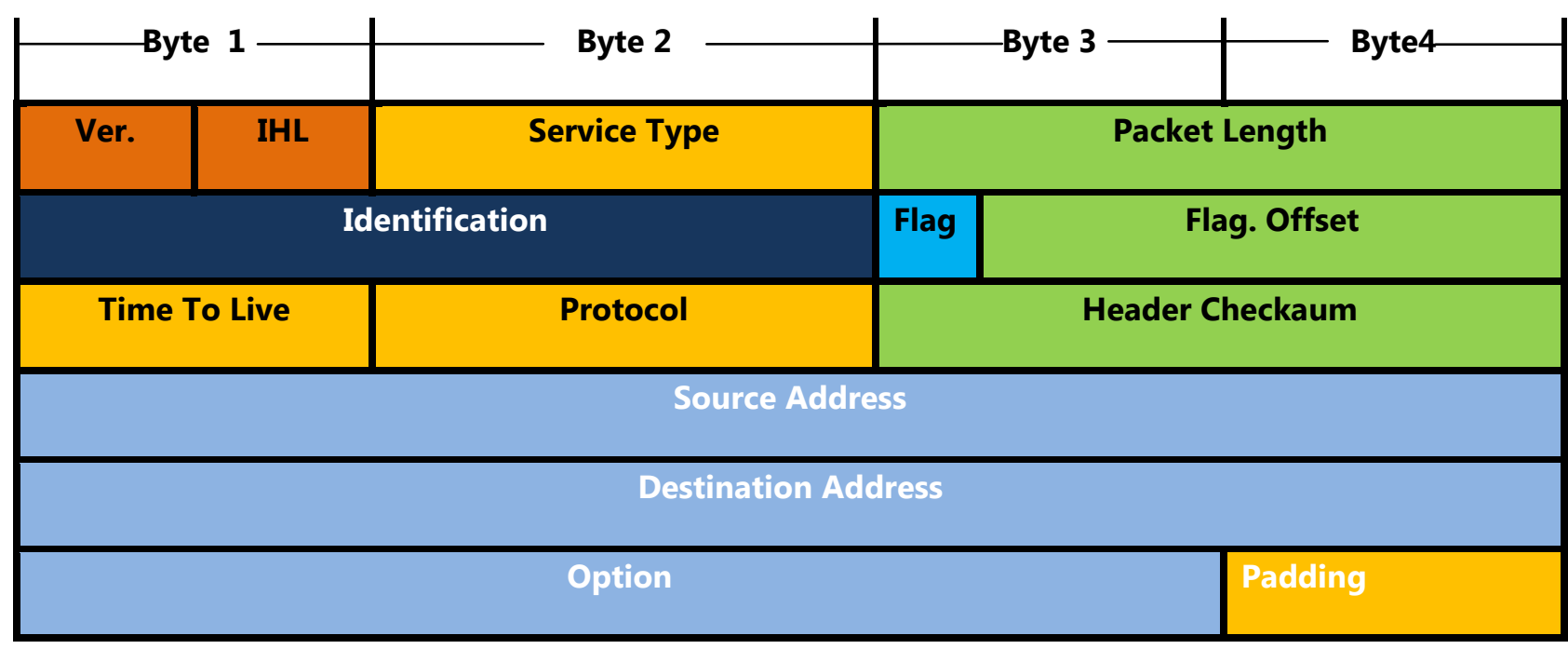

Figure 3 IPv4 Header

\section{Internet Protocol Version6 (IPV6)}

Internet Protocol version 6 (IPv6) is a version of the Internet Protocol (IP) intended to succeed IPv4, which is the protocol currently used to direct almost all Internet traffic. Like IPv4, IPv6 is an internet-layer protocol for packet switched internetworking and provides end-to-end datagram transmission across multiple IP networks. While IPv4 allows 32 bits for an IP address, and therefore has $2^{32}$ (4 294967 296) possible addresses, IPv6 uses 128 -bit addresses, for an address space of 2128 (approximately3.4 $\times 10^{38}$ ) addresses. This expansion allows 
for many more devices and users on the internet as well as extra flexibility in allocating addresses and efficiency for routing traffic. It also eliminates the primary need for network address translation (NAT), which gained widespread deployment as an effort to alleviate IPv4 address exhaustion [12].

One of the goals of IPv6's address space expansion isto make NAT unnecessary, improving total connectivity, reliability, and flexibility. IPv6 will reestablish transparency and end-to-end traffic across the Internet. The new IPv6 addresses are large and cumber some to deal with, so IPv6 reduces the number of people who have to read and write them [13]. A second major goal of IPv6 is to reduce the total time which people have to spend configuring and managing systems. An IPv6 system can participate in "stateless" auto configuration, where it creates a guaranteed-unique IP address by combining its LAN MAC address with a prefix provided by the network router - DHCP is not needed [14].

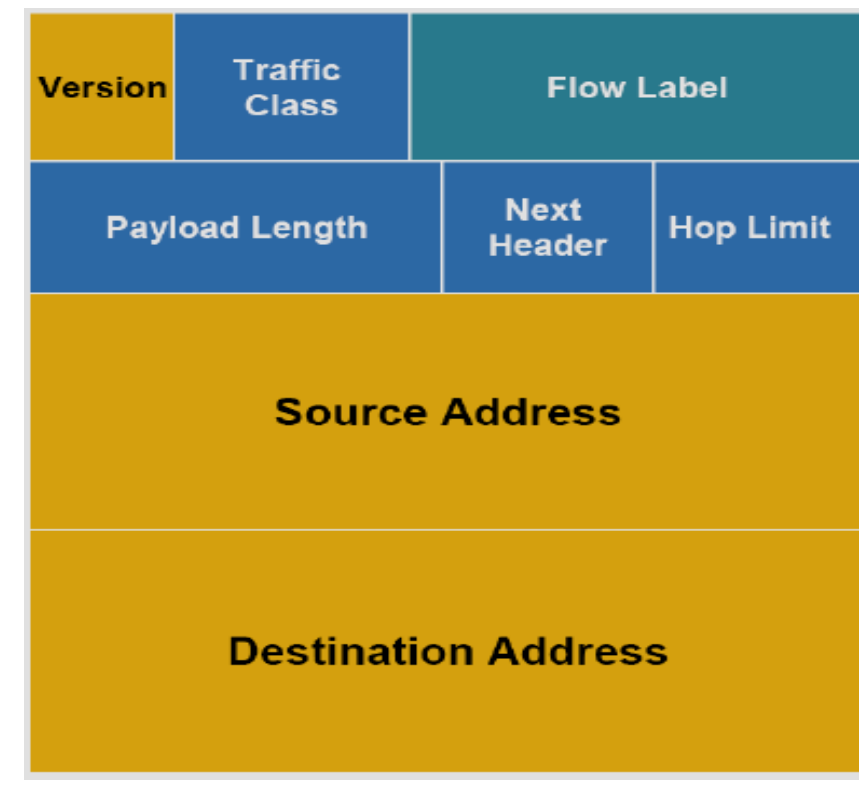

Figure 4 IPv6 Header

\section{Advantages of IPV6}

The main advantage of IPv6 is that this system creates a sufficient number of numbers not only to address the crisis of deferred IPv4 addresses, but also to prevent any future crisis. In the computer industr,In addition, IPv6 addresses will basically be an additional measure, not a revolutionary change in the existing IPv4 system [15]. Nevertheless, for network technicians and other professionals, a number of other features do provide:

- More efficient routing without packet fragmentation;

- Built-in Quality of Service (QoS) technology, which detects delay-sensitive packets;

- Eliminating NAT to expand the address space from 32 to 128 bits;

- Built-in IPsec support (use of IPsec is optional);

- Auto configure addresses to simplify network administration;

- Improved header structure with less processing costs.

Cloud computing is now fundamental to most enterprises, providing cheap, powerful resources such as databases, applications, security and system administration that cannot be afforded individually [16]. IP addresses are critical for orchestrating cloud processes. To commission or decommission cloud virtual machines, multiple IP addresses need to be reserved or freed up with blinding speed. The IPv4-based Internet, increasingly hamstrung by NATs, cannot provide such functionality, and the required numbers of addresses simply do not exist in IPv4.The Internet of Things, the concept of communicating networks of independent devices, is estimated to reach twenty to thirty billion devices by 2020. Every networked device needs an 
address, and IPv4 has a hard limit of 4.3 billion. IPv6 has $340,282,366,920,938,000,000,000,000,000$ billion addresses.

IPv6 is the only technology that can scale to deal with massively distributed cloud infrastructure and the Internet of Things[8] and [17].

Almost all current device operating systems have working IPv6, many with IPv6 enabled by default. See Wikipedia's comparison of IPv6 support in operating systems, and the IPv6 for Microsoft Windows FAQ. There is far more IPv6 traffic on most networks than commonly recognised. If enterprise firewalls have not been expressly configured to handle IPv6, then the enterprise is vulnerable to malicious traffic, no matter how sturdy the old IPv4 defaces are. IPv6 is on by default, and can be accidentally or deliberately used to bypass usage and security policies [18].

\section{Need of IPV6}

Here's the list of indicators that it may be time for you

to consider or integrate IPv6

Your IPv4 network or NAT implementation needs to be fixed or extended.

$>$ You are running out of address space.

> You want to prepare your network for applications that are based on advanced features of IPv6.

$>$ You need end-to-end security for a large number of users and you do not have the address space,

$>$ or you struggle with a NAT implementation.

$>$ Your hardware or applications reach the end of their lifecycle and must be replaced. Make sure

you buy products that support IPv6, even if youdon't enable it right away.

\section{Transition mechanisms of IPV4 AND IPV6}

IPv4 and IPv6 networks are not directly interoperable, which means that a transition mechanism is needed in order to permit hosts on an IPv4 network to communicate with hosts on an IPv6 network, and vice versa. The videos below will help you understand some of these techniques.

That's why some mechanisms where designed:

\section{1- 6in4:}

6in4 is a tunneling technique. You can manually set up a 6 in4 tunnel. Figure 5: Tunneling mechanism

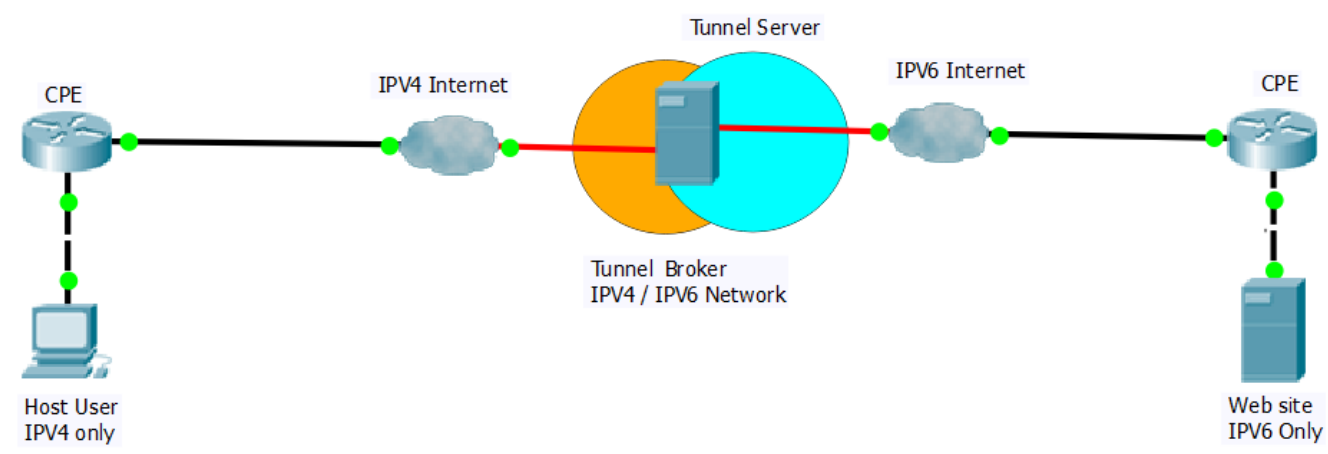

Figure 5: Tunneling mechanism 


\section{2-NAT64}

NAT64 is a transition mechanism based on Network Address Translation (NAT) that makes it possible for IPv6only hosts to talk to IPv4-only servers. NAT64 can be useful for mobile providers.Figure 6:transition mechanism

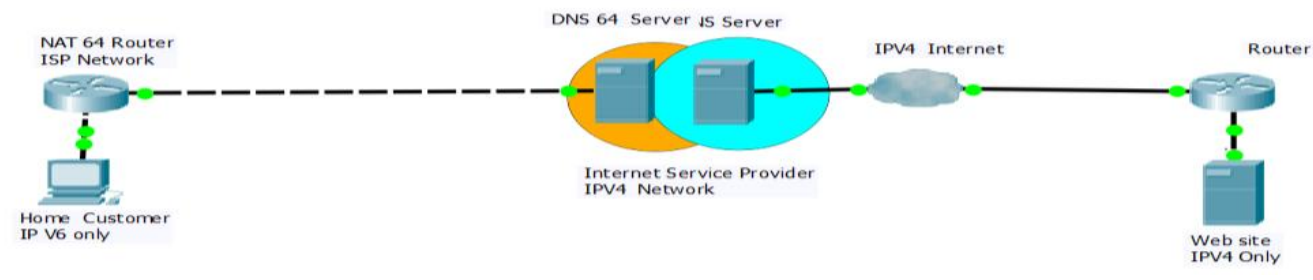

Figure 6:transition mechanism

\section{3- DS-Lite}

DS-Lite allows an ISP to give access to IPv4-only services for customers that have only native IPv6. This mechanism could be useful for DSL or cable providers .Figure 7: Dual stack mechanism

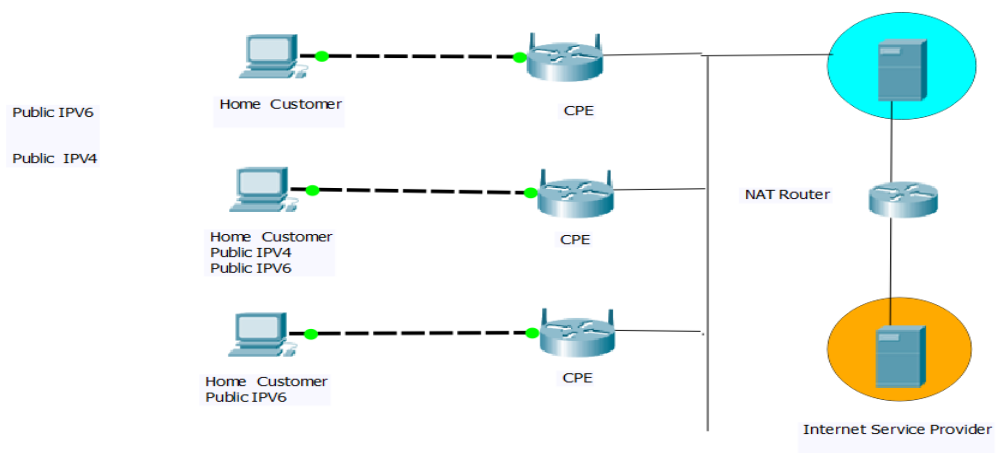

Figure 7:Dual stack mechanism

\section{Comparison between IPV4 ANDIPV6}

IPv6 was designed not to deviate from the existing internet protocol, it was designed to enhance and resolve the problem of the widely deployed protocol, like address space depletion, security. IPV6 also eliminate some feature in IPv4 which is not necessary as an enhancement like NAT technology which violate end to end connection on the internet. Below are some of the performance differences of the two version of protocol difference between IPv6 and IPv4. You can use this table (1)to quickly view summary information, IP functions and how to use IP addresses in Internet IPv4 and IPv6 protocols [19].

Table (1): how to use IP addresses in Internet

\begin{tabular}{|l|l|}
\hline \multicolumn{1}{|c|}{ IPv4 } & \multicolumn{1}{|c|}{ IPv6 } \\
\hline Invented 1981 & Invented 1997 \\
\hline Has 32-bite (4-bytes) address space & Has 128-bite (16-bytes) address space \\
& \\
\hline
\end{tabular}




\begin{tabular}{|c|c|}
\hline IPv4 & IPv6 \\
\hline IPsec is not necessary & IPsec necessary \\
\hline NAT is used & NAT is not used \\
\hline Has a variable length $20-60$ byte & Has a fixed length 40 byte \\
\hline Has 12 field inside the header & Has 8 field inside the header \\
\hline Use Time to Live as field name & Use Hop Limit as field name \\
\hline $\begin{array}{l}\text { Use dotted decimal notation as address format: } \\
\text { 1.0.3.0.6.0.3.0 }\end{array}$ & Use string notation as address format: 1:A:2:4:E:1:3:7 \\
\hline Use $0.0 \cdot 0 \cdot 0.0 .0$ as unknown address & Used :: unknown address \\
\hline It use 127.0.0.1 as loopback address & It use $:: 1$ as loopback address \\
\hline It uses224.0.0.0/4 as Multicast address space & It uses FF00::/8 as Multicast address space \\
\hline Must support DHCP or be configured manually & $\begin{array}{l}\text { Does not require DHCP or manual configuration, it is } \\
\text { auto configuration plug-and-play }\end{array}$ \\
\hline Has broadcast for all the host on the network & $\begin{array}{l}\text { There is no broadcast instead it used group of } \\
\text { multicast }\end{array}$ \\
\hline It does not give data priority & It prioritize data \\
\hline
\end{tabular}




\section{The Migration from IPV4 TO IPV6}

The years from 1997 to 2000 will be characterized by the adoption of IPv6 by ISPs and users. During 1997, users could still have problems related to the newness of products, but starting from 1998, IPv6 will be part of mass-produced protocols distributed on routers, on workstations, and on PCs. At that point, organizations will begin to migrate, less or more gradually, to IPv6. The key goals of the migration are as follow:

- $\quad$ IPv6 and IPv4 hosts must interoperate.

- The use of IPv6 hosts and routers must be distributed over the Internet in a simple and progressive way, with a little interdependence.

- Network administrators and end users must think that the migration is easy to understand and implement.

- A set of mechanisms called SIT (Simple Internet Transition) has been implemented; it includes protocols and management rules to simplify the migration. The main characteristics of SIT are the following:

- Possibility of a progressive and nontraumatic transition: IPv4 hosts and routers can be updated to IPv6, one at a time, without requiring other hosts or routers to be updated simultaneously.

- Minimum requirements for updating: The only requirement for updating hosts to IPv6 is the availability of a DNS server to manage IPv6 addresses. No requirements are needed for routers

- Addressing simplicity: When a router or a host is updated to IPv6, it can also continue to useIPv4addresses

\section{Conclusion}

The current trend in the development of telecommunications networks will be the transition of the Internet to IP version 6 , the obvious advantage of which is the increased address space compared to IPv4. If the IPv4 protocol has $2 \wedge 32$ addresses, then IPv6 has $2^{\wedge} 128$ addresses. IPv 6 contains many and functional improvements, especially in the field of routing. Addressing now has a hierarchical structure, which facilitates the transmission of packets over the network. Also at the IP level there is no more calculation of checksums, which allows routers do not break packets, saving processing time. There were also new QoS and multicast capabilities, and IPSec became mandatory. Maximum size packets in the sixth version of the protocol can reach $4 \mathrm{~GB}$, which will undoubtedly lead to changes in the principles of data transmission in the future .In my experience, we can say that the creation of IPv6 tunnel connections is a viable way to organize the interaction of large networks. At the same time, numerous small networks can use additional means, in particular, automatic tunneling or the 6-to-4 mechanism, which will allow them to connect to the Internet through IPv6 at minimal cost and find an IPv6 provider that suits them. However, based on the results of the work of the experimental network IPv6 Public College, seriously rely on the high quality and reliability of tunnels difficult, if only because one tunnel can hide a long route IPv4, passing through several networks with their specific problems. The most high-quality solution, no doubt, is a real connection to the provider by IPv6.

\section{References:}

[1]Hanumanthappa. J., Manjaiah D H, "A Study on Comparison and Contrast between IPv6 and IPv4 Feature Sets", Conference: International Conference on Computer Networks and Security (ICCNS-2008)

[2] Interface Message Processor. Report No 1822. URL: http://bitsavers.trailing-edge.com/ pdf/bbn/imp/BBN1822_Jan1976.pdf

[3] M.Jothilakshmi,,M.Phil., M.K.Pavithra, S.Yuvabarathi, "Performance Comparison between IPV4 AND IPV6", International Journal of Computer Science Engineering and Technology( IJCSET, 2014. 
[4] TechTarget, 2010. IPv4 or IPv6 - Myths and Realities. URL: http://media.techtarget.com/searchNetworking/downloads/IPv4_or_IPv6.pdf 18.10.2015.

[5] Measuring IPv6 adoption rate and performance in the Internet. Ali EmreYildirim. Oslo University 24.05.2011

[6] Migration from Internet Protocol Version 4 To Internet Protocol Version 6. AmjadMithani. Linneæus University. 2014-11- 24

[7] Wendell Odom; "CCNP ROUTE 642 - 902" Official Certification Guide; 2010; San Francisco, Cisco Press

[8] SiddhartaSaha. URL: http://siddhartasaha.weebly.com/uploads/3/7/7/9/3779388/ipv6_writeup.pdf 18.10.2015

[9] Sharique Ali, Amit Sardar, Kamal Kumar Patra, Rakesh Barua. "Implementation of secure portable real time display system over ethernet network", 3rd International Conference on Recent Advances in Information Technology (RAIT), 2016

[10] Monteiro, Ole Hanseth and Eric Monteiro, 1998. Changing infrastructures: The case of IPv6. URL: http://heim.ifi.uio.no/ oleha/Publications/bok.a.html 18.10.2015

[11] COMPARATIVE ANALYSIS BETWEEN IPv4 AND IPv6. Review Paper vol z(N2),September 2015

[12] URL: http://www.google.com/intl/en/ipv6/statistics.html

[13] RFC 1752: The Recommendation for the IP Next Generation Protocol.

[14] RFC 2462: IPv6 Stateless Address Auto configuration.

[15] RFC 2460: Internet Protocol, Version 6 (IPv6) Specification

[16] RFC 2463: Internet Control Message Protocol (ICMPv6) for the Internet Protocol Version 6 (IPv6).

[17] RFC 3513: Internet Protocol Version 6 (IPv6) Addressing Architecture

[18] John J Amoss, Daniel Minoli 'Handbook of Ipv4 to Ipv6 transition' Auerbach Publications, Newyork,USA.

[19] CiprianPopoviciu, Eric Levy-Abegnoli, Patrick Grossetete 'Deploying IPv6 Networks' Cisco Press, Indianapolis USA, 2006 